\title{
Dynamical energy transfer in ac-driven quantum systems
}

\author{
María Florencia Ludovico,,${ }^{1,}$ Jong Soo Lim, ${ }^{2,3, *}$ Michael Moskalets, ${ }^{4}$ Liliana Arrachea, ${ }^{1}$ and David Sánchez ${ }^{2,5}$ \\ ${ }^{1}$ Departamento de Física, FCEyN, Universidad de Buenos Aires and IFIBA, Pabellón I, Ciudad Universitaria, \\ 1428 CABA, Argentina \\ ${ }^{2}$ Instituto de Física Interdisciplinar y Sistemas Complejos IFISC (UIB-CSIC), E-07122 Palma de Mallorca, Spain \\ ${ }^{3}$ School of Physics, Korea Institute for Advanced Study, Seoul 130-722, Korea \\ ${ }^{4}$ Department of Metal and Semiconductor Physics, NTU “Kharkiv Polytechnic Institute," 61002 Kharkiv, Ukraine \\ ${ }^{5}$ Kavli Institute for Theoretical Physics, University of California, Santa Barbara, California 93106, USA \\ (Received 21 November 2013; revised manuscript received 19 March 2014; published 21 April 2014)
}

\begin{abstract}
We analyze the time-dependent energy and heat flows in a resonant level coupled to a fermionic continuum. The level is periodically forced with an external power source that supplies energy into the system. Based on the tunneling Hamiltonian approach and scattering theory, we discuss the different contributions to the total energy flux. We then derive the appropriate expression for the dynamical dissipation, in accordance with the fundamental principles of thermodynamics. Remarkably, we find that the dissipated heat can be expressed as a Joule law with a universal resistance that is constant at all times.
\end{abstract}

DOI: 10.1103/PhysRevB.89.161306

PACS number(s): 73.23.-b, 72.10.Bg, 73.63.Kv, 44.10.+i

Quite generally, energy flows through a physical system coupled to a power source. In the last decades, typical system sizes have been reduced to the nanoscale and, as a consequence, energy transfer is to be treated quantum mechanically [1]. The fundamental aspects of light-powered biological energy transport [2], thermoelectric waste heat recovery [3], and ultimate refrigeration protocols [4] have been recently uncovered using quantum mechanical principles. However, most discussions are limited to stationary or time-averaged properties [5-9].

Time-dependent quantum transport reveals the dynamical scales that dominate charge transfer across phase-coherent conductors $[10,11]$. A prominent example is the experimentally realized quantum capacitor, which exhibits a pure ac response [12,13]. Applied time-periodic potentials also become a crucial tool to generate directed transport of charge and spin in spatially asymmetric ratchetlike systems $[14,15]$ and to control matter tunneling in Bose-Einstein condensates [16]. Furthermore, the study of ac-driven quantum systems sheds light on the role of fluctuating forces in nanoelectromechanical resonators $[17,18]$. Several aspects related to time-dependent energy transport in electron systems have been also investigated. Heat production in nanoscale engines is discussed in Refs. $[19,20]$ while molecular heat pumping against thermal gradients is proposed in Ref. [21]. Furthermore, the concept of local temperature in ac pumps has been generalized in Ref. [22] whereas universal thermal resistance has been predicted for low-temperature dynamical transport in Ref. [23].

Here, we aim at the time-resolved energy production and redistribution in ac-driven quantum coherent electron systems. We show that the coupling between the different parts of the system not only provides a necessary mechanism for particle exchange, as in the case of charge transport, but also contributes to the energy transport. This contribution is of an

*These authors contributed equally to this work. ac nature. Though the time average of this energy vanishes, it allows for a temporary energy storage. Therefore, the coupling region can be referred to as an energy reactance, which only affects peak power developed in the dynamics. Our goal is also to discuss which portion of the time-resolved energy can be identified as heat, in accordance with the fundamental laws of the thermodynamics.

To be more precise, let us consider a simple but generic model, the resonant level model sketched in Fig. 1. It describes a localized fermion (the impurity) coupled to a fermionic band of continuous density of states (the reservoir). This model has been widely used across disciplines to study asymmetric atomic spectra [24], dissipative quantum mechanics [25], and resonant-tunneling semiconductor heterostructures [26], to name a few. Transitions from the quantum level to the reservoir yield a finite lifetime to the localized fermion which can be represented with a Lorentzian density of states. We consider the case in which the level is attached to a harmonically driven power source as in Fig. 1. Then, the Hamiltonian reads

$$
\mathcal{H}=\mathcal{H}_{C}+\mathcal{H}_{T}+\mathcal{H}_{D}(t)
$$

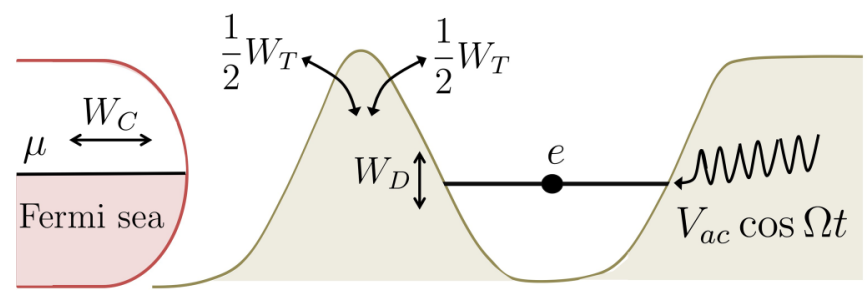

FIG. 1. (Color online) Energy diagram of the system under consideration. A single electronic level (the impurity with charge $e$ ) is coupled to a Fermi sea (the reservoir with chemical potential $\mu$ ). Energy is supplied into the system by a power source (amplitude $V_{\text {ac }}$ and frequency $\Omega$ ) attached to the quantum level. Thus, energy rates are created not only at the impurity $\left(W_{D}\right)$ but also at the reservoir $\left(W_{C}\right)$ and in the contact region $\left(W_{T}\right)$. 
where $\mathcal{H}_{C}=\sum_{k} \varepsilon_{k} c_{k}^{\dagger} c_{k}$ is the continuum of electron states with wave vector $k$ and band energy $\varepsilon_{k}, \mathcal{H}_{T}=\sum_{k}\left(w_{k} d^{\dagger} c_{k}+\right.$ H.c.) describes the tunneling hybridization between propagating electrons and the localized fermion with coupling amplitude $w_{k}$, and $\mathcal{H}_{D}(t)=\varepsilon_{d}(t) d^{\dagger} d$ represents the impurity Hamiltonian with a time-dependent energy level $\varepsilon_{d}(t)=\varepsilon_{0}+$ $V_{\text {ac }} \cos (\Omega t)$, with $\varepsilon_{0}$ being the energy of the bare level. This model can be implemented, e.g., using an electronic terminal coupled to a quantum dot acting as an artificial impurity $[12,13]$ which, in turn, is interacting with a nearby capacitive gate with harmonic driving potential $V_{\mathrm{ac}} \cos (\Omega t)$, where $V_{\mathrm{ac}}$ and $\Omega$ are the ac amplitude and frequency, respectively. Our model is also relevant for fermionic gases of cold atoms [27] in periodically driven optical lattices [28]. For definiteness, we take a single reservoir in the spinless case, but the model can be straightforwardly generalized to account for multiple leads and spinful electrons.

The Hamiltonian given by Eq. (1) conserves the number of particles but not the total energy. We can write

$$
\frac{d\langle\mathcal{H}\rangle}{d t}=W_{C}(t)+W_{T}(t)+W_{D}(t)+P(t),
$$

where the energy fluxes (energy per unit time) are $W_{C}(t)=i\left\langle\left[\mathcal{H}, \mathcal{H}_{C}\right]\right\rangle / \hbar, W_{T}(t)=i\left\langle\left[\mathcal{H}, \mathcal{H}_{T}\right]\right\rangle / \hbar$, and $W_{D}(t)=$ $i\left\langle\left[\mathcal{H}, \mathcal{H}_{D}\right]\right\rangle / \hbar$, and fulfill $W_{C}(t)+W_{T}(t)+W_{D}(t)=0$. The term $P(t)=\left\langle\partial \mathcal{H}_{D} / \partial t\right\rangle$ is the power developed by the ac forces. Importantly, energy transport contains an additional term as compared to charge transport. In the latter case, the current conservation condition reads $I_{C}(t)+I_{D}(t)=0$, where the electronic currents (charge per unit time) in the reservoir and the quantum level are given, respectively, by $I_{C}(t)=$ $i e\left\langle\left[\mathcal{H}, \sum_{k} c_{k}^{\dagger} c_{k}\right]\right\rangle / \hbar$ and $I_{D}(t)=i e\left\langle\left[\mathcal{H}, d^{\dagger} d\right]\right\rangle / \hbar$. There is no particle flux associated with the coupling Hamiltonian $\mathcal{H}_{T}$ (although the currents must, of course, be calculated in the presence of $\mathcal{H}_{T}$ ). In stark contrast, the energy flux in the reservoir, $W_{C}(t)$, cannot be solely inferred from that in the impurity, $W_{D}(t)$, but necessitates knowledge on how energy is absorbed or desorbed in the contact region, $W_{T}(t)$. This crucial fact introduces some ambiguity in the definition of the concept of heat current, as shown below.

The different energy fluxes entering Eq. (2) can be computed in terms of the retarded $\mathcal{G}^{r}\left(t, t^{\prime}\right)=-i \theta(t-$ $\left.t^{\prime}\right)\left\langle\left\{d(t), d^{\dagger}\left(t^{\prime}\right)\right\}\right\rangle$ and lesser $\mathcal{G}^{<}\left(t, t^{\prime}\right)=i\left\langle d^{\dagger}\left(t^{\prime}\right) d(t)\right\rangle$ Green's functions. We find that the energy flux entering the reservoir at time $t$ reads [29]

$$
W_{C}=-2 \operatorname{Re} \int \frac{d \varepsilon}{h} \Gamma(\varepsilon)\left[i \varepsilon \mathcal{G}^{r}(t, \varepsilon) f(\varepsilon)+\mathcal{G}^{<}(t, \varepsilon) \Theta(\varepsilon)\right],
$$

where $\quad \mathcal{G}\left(t, t^{\prime}\right)=\int \frac{d \varepsilon}{2 \pi} e^{-i \varepsilon\left(t-t^{\prime}\right)} \mathcal{G}(t, \varepsilon) \quad$ and $\quad \Theta(\varepsilon)=$ $\int \frac{d \varepsilon^{\prime}}{2 \pi} \frac{\varepsilon^{\prime}}{\varepsilon-\varepsilon^{\prime}-i 0^{+}}$. In Eq. (3), $f(\varepsilon)=1 /\left[1+e^{(\varepsilon-\mu) / k_{B} T}\right]$ is the Fermi-Dirac distribution with background temperature $T$ and chemical potential $\mu$, and $\Gamma(\varepsilon)=2 \pi \sum_{k}\left|w_{k}\right|^{2} \delta\left(\varepsilon-\varepsilon_{k}\right)$ is the resonance width due to coupling to the continuous set of states. For definiteness, we consider a model for the continuum with a flat density of states, corresponding to a constant $\Gamma$. We emphasize that Eq. (3) is completely general and valid to all orders in $\Omega$ and $V_{\text {ac }}$. Moreover, it would be valid even in the presence of Coulomb interactions acting on the spatially localized region.

Following the same procedure, we find for the impurity energy flux the expression

$$
W_{D}(t)=-\varepsilon_{d}(t) I_{C}(t) / e,
$$

where $I_{C}(t)=-(2 e / h) \operatorname{Re} \int d \varepsilon \Gamma(\varepsilon)\left[i \mathcal{G}^{r}(t, \varepsilon) f(\varepsilon)+\mathcal{G}^{<}(t, \varepsilon)\right]$ is the charge current measured in the reservoir. Equation (4) has a rather simple interpretation. Let $n_{d}(t)$ be the expected value of the particle number at the localized site. Then, its total energy rate of change is $d\left[\varepsilon_{d}(t) n_{d}(t)\right] / d t$, which consists of two terms, namely, the ac source power $P(t)=n_{d}(t) d \varepsilon_{d} / d t$ and the energy flux $W_{D}=\varepsilon_{d}(t) d n_{d} / d t=-\varepsilon_{d}(t) I_{C}(t) / e$, since $I_{D}(t) \equiv e d n_{d} / d t=-I_{C}(t)$.

Finally, we determine the energy flux associated with the region that mixes continuous and localized states, $W_{T}=$ $-W_{C}-W_{D}$. It reads

$$
W_{T}(t)=2 \operatorname{Re} \int \frac{d \varepsilon}{h} \partial_{t} \mathcal{G}^{r}(t, \varepsilon) \Gamma f(\varepsilon),
$$

with $\mathcal{G}^{r}(t, \varepsilon)=\sum_{n} e^{-i n \Omega t} \mathcal{G}(n, \varepsilon)$. It is easy to verify that Eq. (5) is a purely ac contribution and vanishes in the limit $\Omega \rightarrow 0$. Thus, for applied static fields or for time-averaged ac transport, this special contribution to the system's energy flow is zero, The quantity $W_{T}$ will be nonzero only for systems exhibiting a dynamical response. In a quantum-dot setup, the tunnel barrier coupling the dot and the reservoir would periodically store and release energy in response to a nearby ac field, thereby the term energy reactance.

To gain further insight into the physical significance of $W_{T}$, we now resort to the scattering-matrix formalism applied to quantum transport. Equivalence between the Green's function and scattering-matrix approaches has been proven in Ref. [30] for averaged time-dependent quantities. But because $W_{T}$ precisely vanishes in the stationary limit, we now analyze the full time-dependent energy flux by considering the energy current density operator $\rho_{E}=\Psi^{*} \mathcal{H} \Psi$, where $\mathcal{H}=$ $-\hbar^{2} \nabla^{2} / 2 m+U(t, \vec{r})$ is the first-quantized version of Eq. (1) and $U$ is the full electronic potential which includes externally applied time-dependent fields. Then, $\rho_{E}$ satisfies the continuity equation [31]

$$
\partial_{t} \rho_{E}+\nabla \cdot W_{E}=S_{E},
$$

where $W_{E}=(\hbar / 4 m i)\left[\Psi^{*} \mathcal{H} \nabla \Psi-\nabla \Psi^{*} \mathcal{H} \Psi+\right.$ H.c. $]$ is the symmetrized energy flux and $S_{E}=\Psi^{*} \partial_{t} U \Psi$ is the source term accounting for the explicit time dependence of $U$. As is customary (see, e.g., Ref. [32]), we introduce the field operator $\hat{\Psi} \sim \int d \varepsilon e^{-i \varepsilon t / \hbar}\left[e^{+i k x} \hat{a}(\varepsilon)+e^{-i k x} \hat{b}(\varepsilon)\right]$ at the cross section $x$ position through which the flux is measured. Then, the energy flux is expressed as

$$
\begin{aligned}
W_{E}(t)= & \sum_{n, q} e^{-i n \Omega t} \int d \varepsilon \frac{\varepsilon_{q}+\varepsilon_{n+q}}{2 h} S^{F *}\left(\varepsilon_{q}, \varepsilon\right) S^{F}\left(\varepsilon_{n+q}, \varepsilon\right) \\
& \times\left[f\left(\varepsilon_{q}\right)-f(\varepsilon)\right],
\end{aligned}
$$

where the Floquet scattering matrix relates the lead outgoing flux operators $\hat{b}$ to the incoming ones $\hat{a}$ via $\hat{b}(\varepsilon)=$ $\sum_{n} S^{F}\left(\varepsilon, \varepsilon_{n}\right) \hat{a}\left(\varepsilon_{n}\right)$ and $\varepsilon_{n}=\varepsilon+n \hbar \Omega$.

If we now insert the generalized Fisher-Lee relation [30,33] $S^{F}\left(\varepsilon_{m}, \varepsilon_{n}\right)=\delta_{m, n}-i \Gamma \mathcal{G}\left(m-n, \varepsilon_{n}\right)$ into Eq. (7) we 
find [29]

$$
W_{E}(t)=W_{C}(t)+\frac{1}{2} W_{T}(t) .
$$

Noticeably, this relation states that in the presence of timedependent fields the energy fluxes entering the reservoir predicted by scattering theory and the Green's function tunneling Hamiltonian approach differ by a term $\frac{1}{2} W_{T}$. Note that this departure occurs for dynamical energy transport only. In the case of time-dependent particle currents or time-averaged energy fluxes the correspondence between the two theoretical frameworks is exact, i.e., $\overline{W_{E}}=\overline{W_{C}}$ with the notation $\overline{(\cdots)}=\int_{0}^{\tau}(\cdots) d t / \tau$, being $\tau=2 \pi / \Omega$. Our results are consistent with discrete models also suggesting that the $\frac{1}{2} W_{T}$ term should be included in the definition of an energy current [34].

What is the origin for the apparent discrepancy in Eq. (8)? Let us turn back to Fig. 1 and examine the role of the contact region. The scattering approach is formulated as a continuum model that considers electrons propagating in a potential landscape $U$ including the reservoirs and the impurity. Alternatively, the resonant level model considers a partition of the energy contributions as in Eq. (1), similarly to Bardeen's picture of tunneling [35]. In addition to both the continuum of states that form the reservoir and the discretized level that constitutes the impurity, this model includes a mixing Hamiltonian $\mathcal{H}_{T}$ which contains creation and annihilation operators associated with electronic degrees of freedom not only in the continuum but also in the localized state. We here emphasize that this part of the system temporarily stores energy which has to be taken into account in order to compare the energy balance with that of a fully continuous model. Equation (8) indicates that a meaningful comparison between both models implies a symmetric splitting of $\mathcal{H}_{T}$, contributing equally to the reservoir and the driven system. Below we provide arguments showing that such splitting is not only natural from the mathematical point of view but also leads to a meaningful definition of heat in the time domain, consistent with the basic laws of thermodynamics.

A concomitant question is which portion of the energy flux can be identified as heat. In stationary systems, where the heat transport is accompanied by the particle transport, the heat flux between the localized system and the reservoir is defined from the change in the energy stored in the reservoir subtracting the convective term originated by the particle flow [36]. Such a definition was also adopted for the dc component of the heat flux in time-dependent driven systems [19], obtaining the same description within the frameworks of the Green's function and scattering-matrix formalisms. However, there is an ambiguity in defining heat in the time domain. This is originated from the fact that $W_{T}(t)$ in the discrete model is nonvanishing in general, although $\bar{W}_{T}=0$. In the scattering approach, such ambiguity is not present because there exists no term as $\mathcal{H}_{T}$. Specifically, Eq. (7) suggests that the appropriate definition is

$$
\dot{Q}(t)=W_{E}(t)-\mu I_{C}(t) / e=W_{C}(t)+\frac{1}{2} W_{T}(t)-\mu I_{C}(t) / e,
$$

while Eq. (2) implies the heat flow definition $\dot{\tilde{Q}}=W_{C}(t)-$ $\mu I_{C}(t) / e$.
We resort to the basic principles of thermodynamics in order to argue that Eq. (9) is the most meaningful definition of heat flux in the time domain. Since the reservoir is a macroscopic system, a suitable interpretation of the different portions of its internal energy under slow variations of the driven localized part would lead to the definition of heat. We proceed as in Ref. [37], identifying as the reservoir the terms of the Hamiltonian $\mathcal{H}$ containing operators $c_{k}^{\dagger}, c_{k}$ and as the driven system those depending on $d^{\dagger}, d$. The tunneling part $\mathcal{H}_{T}$ contains both, hence, it is natural to consider the symmetric splitting $\mathcal{H}_{E}=\mathcal{H}_{C}+\frac{1}{2} \mathcal{H}_{T}$ describing the the reservoir and $\mathcal{H}_{S}(t)=\mathcal{H}_{D}(t)+\frac{1}{2} \mathcal{H}_{T}$ defining the driven system. We then evaluate the rate of change of the internal energy $\left\langle\dot{\mathcal{H}}_{E}\right\rangle=$ $\left\langle\dot{\mathcal{H}}_{C}\right\rangle-\frac{1}{2} \sum_{k}\left[\varepsilon_{k}-\varepsilon_{d}(t)\right] \dot{n}_{k}$, with $n_{k}=\left\langle c_{k}^{\dagger} c_{k}\right\rangle$, which leads us to interpret the quantity $\delta\left\langle\mathcal{H}_{T}\right\rangle=-\sum_{k}\left[\varepsilon_{k}-\varepsilon_{d}(t)\right] \delta n_{k}$ as the chemical work due to particle flow through the contact. Hence, in accordance to the first principle of thermodynamics, an appropriate definition for the heat exchange in the reservoir induced by slow variations of the driven system is $\delta Q=\delta\left\langle\mathcal{H}_{C}\right\rangle+\delta\left\langle\mathcal{H}_{T}\right\rangle / 2-\mu \delta N_{C}$, with $N_{C}=\sum_{k} n_{k}$, as suggested by Eq. (9). In what follows we also show that this expression is also in agreement with the second law of thermodynamics, while this is not the case of the alternative definition $\dot{\tilde{Q}}$.

We focus on the slow-driving regime and consider, for simplicity, zero temperature $(T=0)$. Then, an exact analysis can be performed by means of an expansion in powers of $\Omega$ for the Green's functions (or equivalently of the scattering matrix) [38]:

$$
\mathcal{G}^{r}(t, \varepsilon)=\mathcal{G}_{0}^{r}(t, \varepsilon)+\frac{i \hbar}{2} \partial_{t} \partial_{\varepsilon} \mathcal{G}_{0}^{r}(t, \varepsilon)+\cdots
$$

$\mathcal{G}_{0}^{r}(t, \varepsilon)=\left[\varepsilon-\varepsilon_{d}(t)+i \Gamma / 2\right]^{-1}$ is the frozen Green's function describing the regime in which the electron instantaneously adjusts its potential to the ac field. Considering the expansion of $\mathcal{G}$ up to $O(\Omega)$ yield exact heat fluxes for noninteracting electrons up to $O\left(\Omega^{2}\right)$ [29]. We find $\dot{Q}(t)=\dot{Q}^{(1)}(t)+$ $\dot{Q}^{(2)}(t)$, where the first- and second-order terms in $\Omega$ are, respectively,

$$
\begin{gathered}
\dot{Q}^{(1)}(t)=\int \frac{d \varepsilon}{h}(\mu-\varepsilon) \frac{\partial f}{\partial \varepsilon} \rho_{0}(t, \varepsilon) \frac{d \varepsilon_{d}}{d t}, \\
\dot{Q}^{(2)}(t)=-\frac{1}{2} \int \frac{d \varepsilon}{h} \frac{\partial f}{\partial \varepsilon}\left\{(\mu-\varepsilon) \frac{d}{d t}\left[\left[\rho_{0}(t, \varepsilon)\right]^{2} \frac{d \varepsilon_{d}}{d t}\right]\right. \\
\left.+\left[\rho_{0}(t, \varepsilon) \frac{d \varepsilon_{d}}{d t}\right]^{2}\right\} .
\end{gathered}
$$

Here $\rho_{0}(t, \varepsilon)=-2 \operatorname{Im}\left[\mathcal{G}_{0}^{r}(t, \varepsilon)\right]=\left|\mathcal{G}_{0}^{r}(t, \varepsilon)\right|^{2} \Gamma=-i \partial_{\varepsilon} S_{0} S_{0}^{*}$ is the local density of states and $S_{0}(t, \varepsilon)$ the frozen scattering matrix, i.e., the stationary scattering matrix with time-dependent parameters.

Both the first-order term $\dot{Q}^{(1)}(t)$ and the first term of $\dot{Q}^{(2)}(t)$ vanish at $T=0$ since $-\partial_{\varepsilon} f=\delta(\varepsilon-\mu)$. The component $\dot{Q}^{(2)}(t)$, which is second order in $\Omega$, represents the leading order to the dissipated power in the reservoir. At $T=0$, Eq. (12) reduces to $\dot{Q}^{(2)}(t)=\left[\rho_{0}(t, \mu) \frac{d \varepsilon_{d}}{d t}\right]^{2} / 2$. Evaluating the charge current up to the first order in 


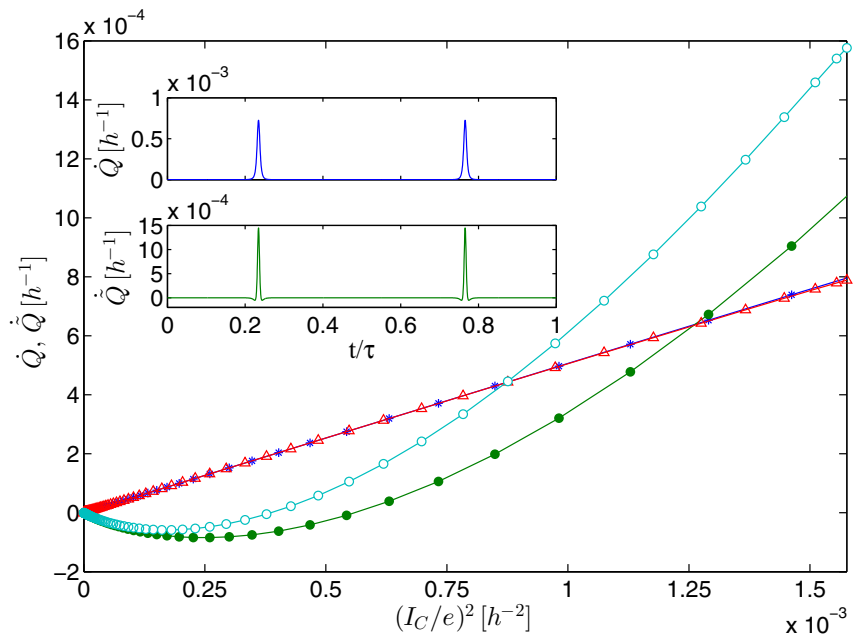

FIG. 2. (Color online) Heat fluxes $\dot{Q}(t)$ (stars and triangles) and $\dot{\tilde{Q}}(t)$ (solid and open circles) as a function of the charge current $I_{C}(t)^{2}$ within the slow-driving regime for two different amplitudes $V_{\mathrm{ac}}=10,12$, respectively. Clearly, only the heat $\dot{Q}(t)$ satisfies $\dot{Q}(t) / I_{C}(t)^{2}=R$, with $R$ a constant independent of time. Parameters: $\mu=0, \varepsilon_{0}=-1.2, T=0$, and $\hbar \Omega=10^{-3}$. Energies are expressed in units of $\Gamma$. Inset: $\dot{Q}(t)$ (dashed lines with a vertical offset) and $\dot{\tilde{Q}}(t)$ (solid lines) as a function of time.

$\Omega$, we find $I_{C}^{(1)}(t)=-(e / h) \rho_{0}(t, \mu) \frac{d \varepsilon_{d}}{d t}$, which implies

$$
\dot{Q}^{(2)}(t)=R_{q}\left[I_{C}^{(1)}(t)\right]^{2},
$$

with $R_{q}=h / 2 e^{2}$ the relaxation resistance quantum [10,11]. Since $R_{q}$ is a manifestly positive quantity at all times, the heat flux given by Eq. (9) represents the heat dissipated into the cold reservoir when the system is coupled to the ac driving force. Therefore, Eq. (9) agrees with the second law of thermodynamics.

We reinforce our conclusion by comparing with the heat rate of change given by $\dot{\tilde{Q}}$. Thus, we evaluate $W_{T}$ up to second order in $\Omega$ :

$$
\begin{aligned}
& W_{T}^{(1)}(t)=2 \int \frac{d \varepsilon}{h} \frac{\partial f}{\partial \varepsilon}\left[\rho_{0}(t, \varepsilon)\left[\varepsilon-\varepsilon_{d}(t)\right] \frac{d \varepsilon_{d}}{d t}\right], \\
& W_{T}^{(2)}(t)=-\int \frac{d \varepsilon}{h} \frac{\partial f}{\partial \varepsilon} \frac{d}{d t}\left[\left[\rho_{0}(t, \varepsilon)\right]^{2}\left[\varepsilon-\varepsilon_{d}(t)\right] \frac{d \varepsilon_{d}}{d t}\right] .
\end{aligned}
$$

Within the weak driving regime, $\dot{\tilde{Q}}(t)=\dot{Q}(t)-\left[W_{T}^{(1)}(t)+\right.$ $\left.W_{T}^{(2)}(t)\right] / 2$, which at $T=0$ contains contributions $\propto \Omega$ and $\propto \Omega^{2}$. Defining the resistance $\tilde{R}(t)$ from the relation $\dot{\tilde{Q}}(t)=\left[I_{C}^{(1)}(t)\right]^{2} \tilde{R}(t)$, we find that $\tilde{R}(t)$ is nonuniversal and depends on time. In fact, it is not even positive definite and then $\dot{\tilde{Q}}(t)$ cannot be interpreted as a dissipated heat. We illustrate in Fig. 2 the behavior of the two expressions of the heat flux for different amplitudes of the driving potential $V_{\text {ac }}$ for a reservoir at $T=0$ and small driving frequencies. The inset shows that, as a function of time, $\dot{Q}(t)$ is always positive whereas $\dot{\tilde{Q}}(t)$ may attain negative values. The main panel shows $\dot{Q}(t)$ and $\dot{\tilde{Q}}(t)$ as a function of $I_{C}(t)^{2}$ within the slow-driving regime. In the first case, we observe a linear function with the universal slope $R_{q}$. In contrast, in the second case we observe a nonuniversal behavior, including negative values of $\tilde{R}(t)$. The two definitions of heat, however, lead to the same result when averaged in time, $\overline{\dot{Q}}=\overline{\dot{\tilde{Q}}}=\bar{P}$, and, therefore, only a pure dynamical measurement would be able to distinguish both.

In conclusion, we have discussed the dynamical heat generation in a resonant level system due to coupling to an external time-dependent potential and highlighted the important role played by the energy associated with the coupling region. The latter is unique to dynamical energy transport. By recourse to an adiabatic expansion valid for the slow-driving regime, we have found that an appropriate expression of the dynamical heat flux consistent with the fundamental principles of thermodynamics requires to take into account the work associated with particles flowing through the tunneling region. Importantly, we have shown that properly taking into account this contribution to the total energy leads to a complete agreement between the scattering-matrix and Green's function approaches for continuum and tunneling models, respectively. A naive extension of the dc heat flux expression for the tunneling model would lead to incorrect results in the time domain. Furthermore, we have found that the time-dependent heat current is instantaneously given by a Joule law with a universal resistance. This is an experimentally testable prediction which is relevant in view of recent developments that emphasize the energetics of atomic systems and nanostructures.

We acknowledge M. Büttiker and R. López for numerous discussions. This work was supported in part by MINECO Grants No. FIS2011-23526 and No. CSD2007-00042 (CPAN), the NSF under Grant No. NSF PHY11-25915, the UBACyT, CONICET, and MINCyT, Argentina.
[1] S. Jezouin, F. D. Parmentier, A. Anthore, U. Gennser, A. Cavanna, Y. Jin, and F. Pierre, Science 342, 601 (2013).

[2] N. Lambert, Y. N. Chen, Y. C. Cheng, C. M. Li, G. Y. Chen, and F. Nori, Nat. Phys. 9, 10 (2013).

[3] L. D. Hicks and M. S. Dresselhaus, Phys. Rev. B 47, 16631 (1993).

[4] F. Giazotto, T. T. Heikkilä, A. Luukanen, A. M. Savin, and J. Pekola, Rev. Mod. Phys. 78, 217 (2006).

[5] F. Giazotto and M. J. Martínez-Pérez, Nature (London) 492, 401 (2012).
[6] W. Lee, K. Kim, W. Jeong, L. A. Zotti, F. Pauly, J. C. Cuevas, and P. Reddy, Nature (London) 498, 209 (2013).

[7] B. Sothmann, R. Sánchez, A. N. Jordan, and M. Büttiker, New J. Phys. 15, 095021 (2013).

[8] J. V. Koski, T. Sagawa, O.-P. Saira, Y. Yoon, A. Kutvonen, P. Solinas, M. Möttönen, T. Ala-Nissila, and J. P. Pekola, Nat. Phys. 9, 644 (2013).

[9] J.-P. Brantut, C. Grenier, J. Meineke, D. Stadler, S. Krinner, C. Kollath, T. Esslinger, and A. Georges, Science 342, 713 (2013). 
[10] M. Büttiker, A. Prêtre, and H. Thomas, Phys. Lett. A 180, 364 (1993).

[11] M. Büttiker, A. Prêtre, and H. Thomas, Phys. Rev. Lett. 70, 4114 (1993).

[12] J. Gabelli, G. Fève, J.-M. Berroir, B. Plaçais, A. Cavanna, B. Etienne, Y. Jin, and D. C. Glattli, Science 313, 499 (2006).

[13] G. Fève, A. Mahé A, J. M. Berroirm, T. Kontos, B. Plaçais, D. C. Glattli, A. Cavanna, B. Etienne, and Y. Jin, Science 316, 1169 (2007).

[14] H. Linke, T. E. Humphrey, A. Löfgren, A. Sushkov, R. Newbury, R. P. Taylor, and P. Omling, Science 286, 2314 (1999).

[15] M. V. Costache and S. O. Valenzuela, Science 330, 1645 (2010).

[16] H. Lignier, C. Sias, D. Ciampini, Y. Singh, A. Zenesini, O. Morsch, and E. Arimondo, Phys. Rev. Lett. 99, 220403 (2007).

[17] G. A. Steele, A. K. Hüttel, B. Witkamp, M. Poot, H. B. Meerwaldt, L. P. Kouwenhoven, and H. S. J. van der Zant, Science 325, 1103 (2009).

[18] B. Lassagne, Y. Tarakanov, J. Kinaret, D. Garcia-Sanchez, and A. Bachtold, Science 325, 1107 (2009).

[19] L. Arrachea, M. Moskalets, and L. Martin-Moreno, Phys. Rev. B 75, 245420 (2007).

[20] M. Moskalets and M. Büttiker, Phys. Rev. B 80, 081302(R) (2009).

[21] D. Segal and A. Nitzan, Phys. Rev. E 73, 026109 (2006).

[22] A. Caso, L. Arrachea, and G. S. Lozano, Phys. Rev. B 83, 165419 (2011).
[23] J. S. Lim, R. López, and D. Sánchez, Phys. Rev. B 88, 201304(R) (2013).

[24] U. Fano, Phys. Rev. 124, 1866 (1961).

[25] F. Guinea, V. Hakim, and A. Muramatsu, Phys. Rev. B 32, 4410 (1985).

[26] A.-P. Jauho, N. S. Wingreen, and Y. Meir, Phys. Rev. B 50, 5528 (1994).

[27] J.-P. Brantut, J. Meineke, D. Stadler, S. Krinner, and T. Esslinger, Science 337, 1069 (2012).

[28] T. Salber, S. Kling, T. Hecking, C. Geckeler, L. Morales-Molina, and M. Weitz, Science 326, 1241 (2009).

[29] See Supplemental Material at http://link.aps.org/supplemental/ 10.1103/PhysRevB.89.161306 for the complete derivation.

[30] L. Arrachea and M. Moskalets, Phys. Rev. B 74, 245322 (2006).

[31] W. N. Mathews, Jr., Am. J. Phys. 42, 214 (1974).

[32] M. V. Moskalets, Scattering Matrix Approach to Non-Stationary Quantum Transport (World Scientific, London, 2012).

[33] D. S. Fisher and P. A. Lee, Phys. Rev. B 23, 6851 (1981).

[34] L.-A. Wu and D. Segal, J. Phys. A: Math. Theor. 42, 025302 (2009).

[35] J. Bardeen, Phys. Rev. Lett. 6, 57 (1961).

[36] G. D. Mahan, Many-Particle Physics (Plenum, New York, 2000).

[37] R. Balian, From Microphysics to Macrophysics, Vol. I (Springer, Berlin, 2007).

[38] M. Moskalets and M. Büttiker, Phys. Rev. B 69, 205316 (2004). 